\title{
TWO-DIMENSIONAL SOAP FROTHS AND POLYCRYSTALLINE NETWORKS: WHY ARE LARGE CELLS MANY-SIDED?
}

\author{
CW J BEENAKKER \\ Philps Rescarch Laboratortes $5600 \mathrm{JA}$ Endhoicn The Netherlands
}

\begin{abstract}
The correlation between the ared of a cell and its number of sides found in coasening two dimensional networks (soap froths and polyeiystallne materials) is studied both analytically and by numerical simulation This shape-size correlation is explaned as a dynamical consequence of the shape-dependent growth rate of the cells
\end{abstract}

\section{Introduction}

The question posed in the title refers to a curious shape-size correlation found in certain two-dimensional (2D) networks ${ }^{i}$ ) There is no apparent geometrical reason why large cells should have many sides, and yet this correlation is observed in such diverse systems as biological tissue ${ }^{2}$ ), soap froths $^{3}$ ), and polycrystalline metals and ceramıcs ${ }^{+}$) Empirical rules have been proposed to describe the corrclation in the different systems, known as Lewis' law $\left.^{2}\right) \bar{A}(n) \propto n+$ constant, and the perimeter law $\left.{ }^{4}\right) \vee V \bar{A}(n) \propto n+$ constant (We denote by $\bar{A}(n)$ the average area of $n$-sided cells) The ubiquity of the shape-size correlation led Rivier ${ }^{5}$ ) to argue that the empirical rules hold because they maximize the network entropy (for different sets of constraints) His argument is certainly general, but leaves unanswered the obvious question Why maximize the entropy in these non-equilibrium systems?

We have no intention of proving or disproving the maximum entiopy postulate Our aim is simply to deduce the mechanism of the correlation in a ceitain class of systems from the equations of motion describing their approach to equilibrium We shall have nothing to say about the biological systems, but restrict ourselves to the soap froths and polycrystalline materials These have in common that their dynamics is governed by surface tension trying to shorten the cell boundaries The equations of motion for this coarsenıng process take an especially simple form in 2D, see section 2 A remarkable property of thesc equations is that many-sided cells grow, while few-sided cells shrink ${ }^{67}$ ) We propose that the observed correlation between $A$ and $n$ is a dynamical 
consequence of the dependence of $\mathrm{d} A / \mathrm{d} t$ on $n$. An analytic calculation in section 3 , based on a highly simplified version of the model of section 2 , shows that indeed this shape-dependent growth rate leads in the long-time "scaling" regime to shape-size correlations of the type observed. The dependence of $\bar{A}(n)$ on $n$ turns out not to be fully described by either Lewis' law or the perimeter law, but is of a more complicated form. A numerical simulation ${ }^{8}$ ) of the full model bears out these general conclusions, as shown in section 4. Our results are compared with a recent experiment* on a quasi-2D soap froth by Glazier et al. $^{3}$ ), and with a lattice model simulation by Sahni et al. $^{4}$ ) of $2 \mathrm{D}$ grain growth in polycrystalline materials. (A direct comparison with experiment for the latter case is not made, since only data for $3 \mathrm{D}$ grain growth is available.) Section 4 also contains a comparison with mean-field calculations by Marder ${ }^{12}$ ). We conclude in section 5 with some final remarks.

\section{Equations of motion}

We consider a 2D network with three-fold coordination of the vertices, see fig. 1. The equations which govern the motion of the boundaries are different for a polycrystalline network (P) and a soap froth (S). In case $\mathrm{P}$ the motion of the grain boundaries (which separate crystallites of different orientation) can

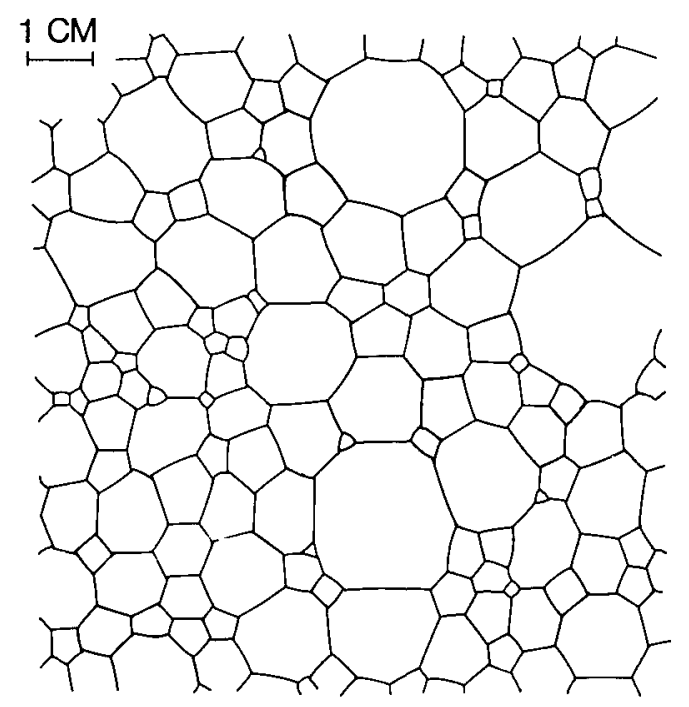

Fig 1 Two-dimensional soap film network, traced from an experimental photograph made by C S Smith (icf 9) The froth lies between parallel glass plates, spaced about $4 \mathrm{~mm}$ apart.

${ }^{4}$ Earlier cxpcriments did not show a scalıng regıme ${ }^{9}$ ), sce section 5 
be described by the curvature rule ${ }^{13}$ ) $V=m \Gamma$ ( $V$ is the normal velocity component, $\Gamma$ the local curvature of the boundary, and $m$ a mobility coefficient). In case $\mathrm{S}$ we have instead a linear relation between the flux of gas $\Psi$ through the soap film and the pressure difference $\Delta p$ between adjacent cells, $\Psi=\mu \Delta p$ ( $\mu$ is a permeability coefficient). Laplace's law $\Delta p=\sigma \Gamma$ (with surface tension coefficient $\sigma$ ) then implies $\Psi=\mu \sigma \Gamma$. Moreover, the soap film must have a uniform curvature (since there is a uniform pressure inside a cell), so that soap-cell boundaries are circular arcs - in contrast to grain boundaries which have more irregular shapes.

In two dimensions a reduced description of the dynamics is possible, which allows us to treat both cases $\mathrm{P}$ and $\mathrm{S}$ on the same basis. The reduction is obtained by calculating the time derivative of the area $A$ of a cell in the network

$$
\frac{\mathrm{d} A}{\mathrm{~d} t}=- \text { constant } \times \oint \mathrm{d} l \Gamma
$$

where the constant equals $m$ in case $\mathrm{P}$, and $\mu \sigma$ in case $\mathrm{S}$ (an incompressible gas is assumed). The line integral of the curvature is taken along the perimeter of the cell and can be evaluated by inserting the definition $\Gamma \equiv \mathrm{d} \phi / \mathrm{d} l$ ( $\phi$ is the polar angle of the tangent to the curve). The result may be written as

$$
\oint \mathrm{d} l \Gamma=2 \pi-\sum_{i=1}^{n}\left(\pi-\alpha_{t}\right),
$$

where $\alpha_{1}, \ldots, \alpha_{n}$ are the internal angles at the $n$ vertices of the cell. Local equilibrium requires that in the infinitesimal region of intersection the three boundaries meet at equal angles of $120^{\circ}$. Putting $\alpha_{t}=2 \pi / 3$ in eq. (2.2) and combining with eq. (2.1), one then obtains the evolution equation

$$
\frac{\mathrm{d} A}{\mathrm{~d} t}=k(n-6),
$$

with $k=(\pi / 3) m$ in case $\mathrm{P}$, and $(\pi / 3) \mu \sigma$ in case $\mathrm{S}$. This remarkable equation is known as von Neumann's theorem ${ }^{6}$ ) in the case of a soap froth, or as the area theorem $\mathrm{m}^{7}$ ) in the metallurgical context. Supplemented by a simple model for changes in $n$ (see below), it allows a reduced description of the coarsening process in terms of only two variables $A$ and $n$ per cell. In this description the different form of the boundaries in cases $\mathrm{P}$ and $\mathrm{S}$ does not enter.

Before proceeding with a discussion of the mechanisms by which a cell can change its number of sides, we note one important consequence of eq. (2.3). Since the total area of the $N$ cells is conserved (for an infinite system), we have 


$$
\frac{\mathrm{d}}{\mathrm{d} t} \sum_{j=1}^{N(t)} A_{j}(t)=0
$$

which implies*

$$
\sum_{j=1}^{N(t)}\left(n_{J}-6\right)=0 .
$$

That the cells have 6 sides on average may also be derived from Euler's theorem, cf. ref. 1 .

Marder ${ }^{12}$ ) has proposed a very simple way to model the dynamics of $n$, which goes as follows. In principle, a cell can change its number of sides by either ${ }^{1}$ ) a neighbour-switching "T1-process", or by a "T2-process" involving the disappearance of a cell. This is illustrated in fig. 2. In the T1-process two cells lose and two cells gain a side. In the T2-process the number of sides gained or lost depends on whether the disappearing cell has 3,4 , or $5 \operatorname{sides}^{\mathrm{r}}$ : If a 3-sided cell disappears, each of its neighbours loses a side; if the disappearing cell has either 4 or 5 sides, two neighbours lose a side - and in addition in the latter case one other neighbour gains a side. Note that as a result of each of these elementary processes the average number of sides of the cells remains 6 , as it should. In the experiments of Glazier et al. ${ }^{3}$ ) it is observed that in a coarsening soap froth only a small fraction of the topological changes
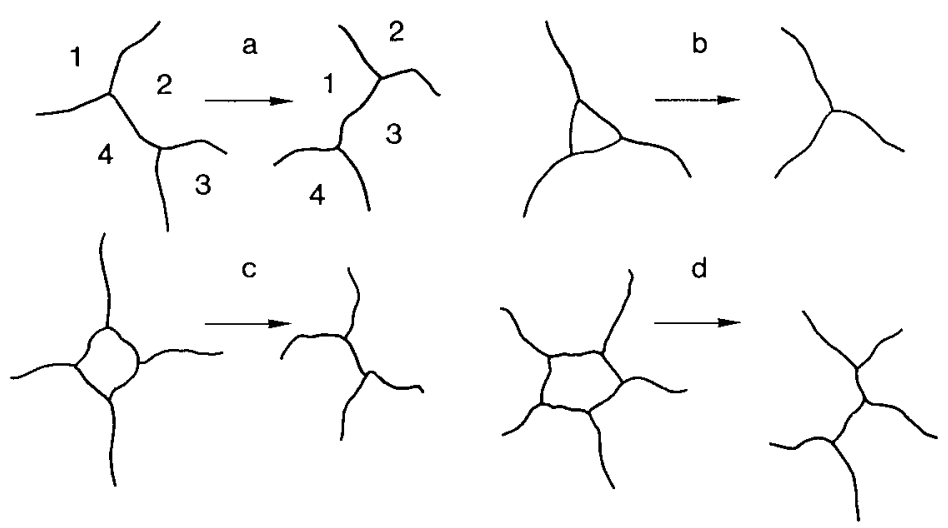

Fig 2 Sketch of grain boundaries undergong topological changes Fig 2d shows the T1- or neighbour-switching process, figs $2 \mathrm{~b}-\mathrm{d}$ show $\mathrm{T} 2$-processes related to a disappcarıng 3-, 4-, or 5 -sided grain (Notice how in Fig 2d one gran ends up ganıng a side)

"The time derivative and the summation in eq (24) may be interchanged, since cells which disappear necessarily have a vanishing ared and therefore do not contribute to the sum

**When a 2-sided cell disappears, each of its neighbours loses two sides These lense-shaped cells are very rare 
occurs via neighbour switching without a disappearıng cell We shall assume that these T1-processes play an unimportant role in grain growth as well, and may be neglected The description of the dynamics of $n$ is now complete, but for one final point When a 4- or 5-sided cell disappedrs one must specify which two (mutually non-adjacent) neighbours lose a side in the T2-process, cf fig $2 \mathrm{c}, \mathrm{d}$ We shall assume that each such pair is equivalent and choose it at random* This seems reasonable in view of the equi axed form of the cells Marder ${ }^{12}$ ), on the contrary, imposes the bias that the two smallest neighbours always lose a side We shall return to this point in section 4

In ref 8 we have described how the codrsening of a $2 \mathrm{D}$ network can be simulated on a computer by means of the above model A "mean-field" version, in which correlations between different cells are neglected, has been studied by Marder ${ }^{17}$ ) We defer a discussion of these numerical results to section 4 First, a qualitative analytic treatment is given, which explicitly brings out the dynamical onigin of the shape-size correlation

\section{Qualitative theory}

The main features of the correlation between area and number of sides may be obtained analytically from a simplified version of the model of section 2 In this simplification the deterministic dynamics of the number of sides $n$ is replaced by a diffusion process for a continuous variable $\nu$, so that the distribution function $P(A, \nu, t)$ evolves in time according to

$$
\begin{aligned}
\frac{\partial}{\partial t} P(A, \nu, t)= & -k \frac{\partial}{\partial A}(\nu-6) P(A, \nu, t)-\frac{\partial}{\partial \nu} \Omega_{1}(t) P(A, \nu, t) \\
& +\frac{1}{2} \frac{\partial^{2}}{\partial \nu^{2}} \Omega_{2}(t) P(A, \nu, t)
\end{aligned}
$$

The first term on the $\mathrm{r}$ h s corresponds to von Neumann's theorem, eq (2 3 ) The remaining two terms describe the diffusion process, with drift and diffusion coefficients $\Omega_{1}$ and $\Omega_{2}$ These terms are consistent with Marder's ${ }^{12}$ ) mean field equation in the continuum approximation, if we identify $\Omega_{1}=$ $\Omega_{+}-\Omega_{-}$, and $\Omega_{2}=\Omega_{+}+\Omega_{-}$, where $\Omega_{+}$and $\Omega$ are the probabilities per unit time that a cell gains, respectively loses a side Note that, since $\Omega_{ \pm}>0$, this identification implies $\Omega_{\rho}>\left|\Omega_{1}\right|$ For simplicity we ignore here any dependence of the $\Omega$ 's on $A$ and $\nu$ (see ref 12 for a more realistic choice of $\Omega$ ) Eq (3 1) is

*There is onc rare exception If as a result of our tandom choice a spuious boundary would appear (i e a boundary which does not separate two distinct cells) then another pair is chosen In particular a 2 sided cell is not allowed to lose a side 
supplemented by the boundary condition

$$
P(0, \nu, t)=0 \quad \text { for } \nu>6,
$$

which expresses the fact that grains can vanish (if $\nu<6$ ) but not reappear. In addition one might include a reflective boundary at $\nu=2$, to ensure that cells do not have less than 2 sides. This is not essential to the problem, and is omitted here, since anyway cells with $\nu<2$ shrink rapidly and disappear.

Numerical simulation ${ }^{8}$ ) has shown that, after transients have died away, the system enters a scaling regime in which the normalized distribution function depends on time only through the average area $\bar{A}(t)$ - which itself increases linearly with $t$. Let us look for a solution of this form, thereby restricting ourselves to the long-time regime. We thus substitute into eq. (3.1) the Ansatz

$$
\begin{aligned}
& P(A, \nu, t)=N(t) \bar{A}(t)^{-1} p(A / \bar{A}(t), \nu), \\
& \bar{A}(t)=\frac{1}{\gamma} k t
\end{aligned}
$$

with $\gamma$ an undetermined numerical coefficient. The prefactor $N / \bar{A}$ in eq. (3.3a) ensures that $p$ is normalized to unity,

$$
\int_{0}^{\infty} \mathrm{d} a \int_{-\infty}^{\infty} \mathrm{d} \nu p(a, \nu)=1, \text { with } a \equiv A / \bar{A} .
$$

The resulting equation is

$$
\begin{array}{rl}
-2 & p(a, \nu)+[\gamma(\nu-6)-a] \frac{\partial}{\partial a} p(a, \nu) \\
=-t \Omega_{1}(t) \frac{\partial}{\partial \nu} p(a, \nu)+\frac{1}{2} t \Omega_{2}(t) \frac{\partial^{2}}{\partial \nu^{2}} p(a, \nu),
\end{array}
$$

where we have used that the total area $N \bar{A}$ is constant in time. The r.h.s. of eq. (3.4) is time independent provided $\Omega_{1}$ and $\Omega_{2}$ scale as $1 / t$ in the long-time limit, so that $t \Omega_{1,2}(t) \equiv \omega_{1,2}=$ constant.

We are now in the position to analyze the correlation between area and number of sides which has developed in the scaling regime. To this end we take the first two moments of $p(a, \nu)$ with respect to $a$,

$$
p(\nu) \equiv \int_{0}^{\infty} \mathrm{d} a p(a, \nu) \quad \text { and } \quad \bar{a}(\nu) \equiv p(\nu)^{-1} \int_{0}^{\infty} \mathrm{d} a a p(a, \nu),
$$


which according to eq. (3.4) satisfy

$$
\begin{aligned}
& \frac{1}{2} \omega_{2} \frac{\mathrm{d}^{2}}{\mathrm{~d} \nu^{2}} p(\nu)-\omega_{1} \frac{\mathrm{d}}{\mathrm{d} \nu} p(\nu)+p(\nu)=-\gamma(\nu-6) p(a=0, \nu), \\
& \frac{1}{2} \omega_{2} \frac{\mathrm{d}^{2}}{\mathrm{~d} \nu^{2}}[p(\nu) \bar{a}(\nu)]-\omega_{1} \frac{\mathrm{d}}{\mathrm{d} \nu}[p(\nu) \bar{a}(\nu)]=-\gamma(\nu-6) p(\nu) .
\end{aligned}
$$

To close these two equations we need a guess for $p(0, \nu)$. This function is zero for $\nu>6$ (eq. (3.2)) and rapidly decreases to zero for $\nu<2$, with a peak in between. The simplest way to model this behavior is by a delta function, say at $\nu=3$. Substituting $p(0, \nu)=$ constant $\times \delta(\nu-3)$ into eq. (3.5), and eliminating the constant by requiring that $p(\nu)$ is normalized to unity, we obtain

$$
\frac{1}{2} \omega_{2} \frac{\mathrm{d}^{2}}{\mathrm{~d} \nu^{2}} p(\nu)-\omega_{1} \frac{\mathrm{d}}{\mathrm{d} \nu} p(\nu)+p(\nu)=\delta(\nu-3) .
$$

At this point we note that the value of $\omega_{1}$ is completely determined by eqs. (3.6) and (3.7). Indeed, multiplying both sides of eq. (3.7) by $\nu$ and integrating we find that

$$
\omega_{1}=3-\int_{-\infty}^{\infty} \mathrm{d} \nu \nu p(\nu)=-3,
$$

where we have used that the average of $\nu$ equals 6 (this follows from eq. (3.6) upon integration over $\nu$ ).

The differential equations (3.6) and (3.7) can be solved by elementary methods (cf. ref. 14). The results are

$$
\begin{aligned}
& p(\nu)=\frac{2}{\omega_{2} \Delta \lambda}\left(\mathrm{e}^{-\lambda_{-}(\nu-3)}-\mathrm{e}^{-\lambda_{+}(\nu-3)}\right) \theta(\nu-3), \\
& \frac{1}{\gamma} \bar{a}(\nu)=\nu-6+\frac{1}{2} \omega_{2} \Delta \lambda+\omega_{2} \Delta \lambda \frac{\mathrm{e}^{-\Delta \lambda(\nu-3)}-\mathrm{e}^{-\lambda_{+}(\nu-3)}}{1-\mathrm{e}^{-\Delta \lambda(\nu-3)}}, \text { for } \nu>3,
\end{aligned}
$$

where we have defined

$$
\lambda_{ \pm} \equiv \frac{3}{\omega_{2}}\left[1 \pm\left(1-\frac{2}{9} \omega_{2}\right)^{1 / 2}\right] \text { and } \Delta \lambda \equiv \lambda_{+}-\lambda_{-} .
$$

The function $\theta(\nu-3)$ in eq. (3.9) is 1 for $\nu>3$ and 0 for $\nu<3$. The requirement of real eigenvalues $\lambda_{ \pm}$, necessary for a non-negative $p$, limits the 


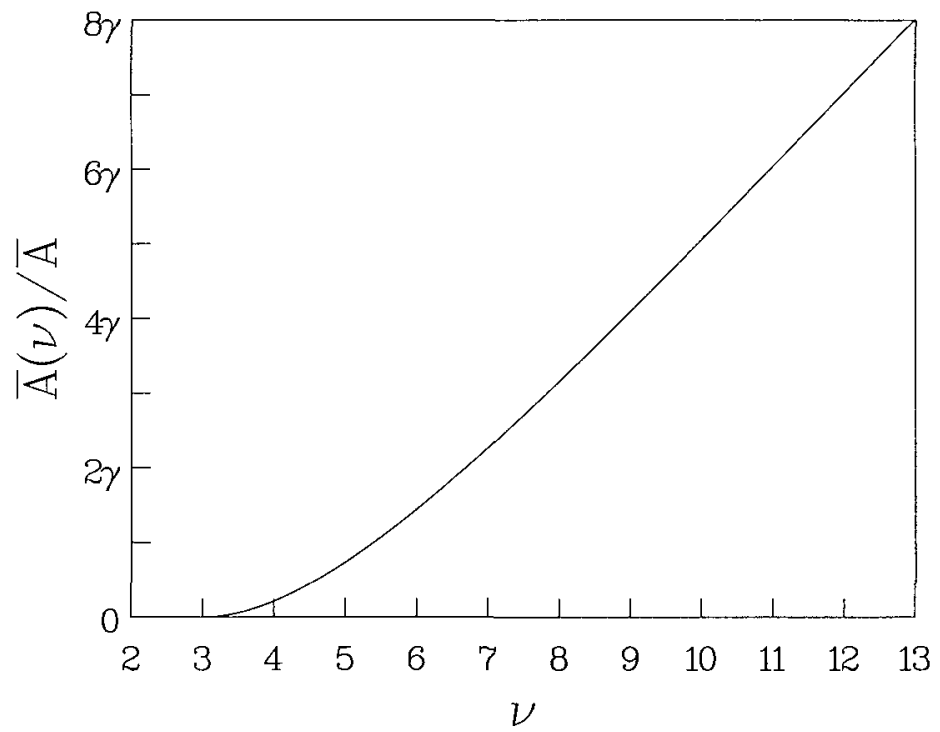

Fig 3 Scalcd average area versus number of sides, according to eq (3 10) (Vertical axis in units of the dimensionless coarsening rate coefficient $\gamma$ ) This is the result of the qualitative theory, in which the number of sides is treated as a continuous variable $\nu$ Shown is the curve for $\omega_{2}=4$, other values of $\omega_{2}$ in the range (3 11) give similar results

allowed values of the parameter $\omega_{2}$ to $\omega_{2}<\frac{9}{2}$. Together with the previous restriction $\omega_{2}>\left|\omega_{1}\right|$ this gives

$$
3<\omega_{2}<\frac{9}{2} \text {. }
$$

Both $p(\nu)$ and $\bar{a}(\nu)$ are not very sensitive to variations of $\omega_{2}$ in this range, so that eq. (3.10) essentially determines the scaled average area $\bar{a}(\nu) \equiv \bar{A}(\nu) / \bar{A}$ up to the multiplicative coefficient $\gamma$.

As shown in fig. 3 , a positive correlation between area and number of sides is found. The average area as a function of the number of sides increases rather slowly for few-sided cells, and then crosses over to a more rapid linear increase for many-sided cells. These qualitative features are indeed observed both experimentally and in numerical simulations, as we shall now discuss.

\section{Numerical results}

We have studied the model of section 2 by a method of numerical simulation described previously ${ }^{8}$ ). The system initially consists of over $10^{5}$ cells, with a 
broad distribution of cell areas and number of sides '. In fig. 4 we have plotted the ratio $\bar{A}(n) / \bar{A}$ versus $n$. This quantity is time independent in the scaling regime. Our numerical results (solid curve) confirm the main features of the shape-size correlation found in the qualitative theory of the previous section: As in fig. 3, a positive correlation is seen between $A$ and $n$, characterized by an inital slow increase of $\bar{A}(n)$ with $n$ and a more rapid increase for $n>6$. The data also seems to confirm the linear dependence for large $n$ (although with a smaller slope than predicted ${ }^{k \star}$ ). We do not, however, consider our numerical data conclusive evidence for the asymptotic linear law, in view of the statistical uncertainties at the two largest values of $n$. Note that Marder's mean-field calculation $^{12}$ ) gives a quite different large- $n$ behaviour (dashed curve). It would be interesting to know if the difference is due to Marder's neglect of correlations or to the bias in his dynamical rules (which, as mentioned in section 2, causes small cells to have a greater probability to lose a side than large cells).

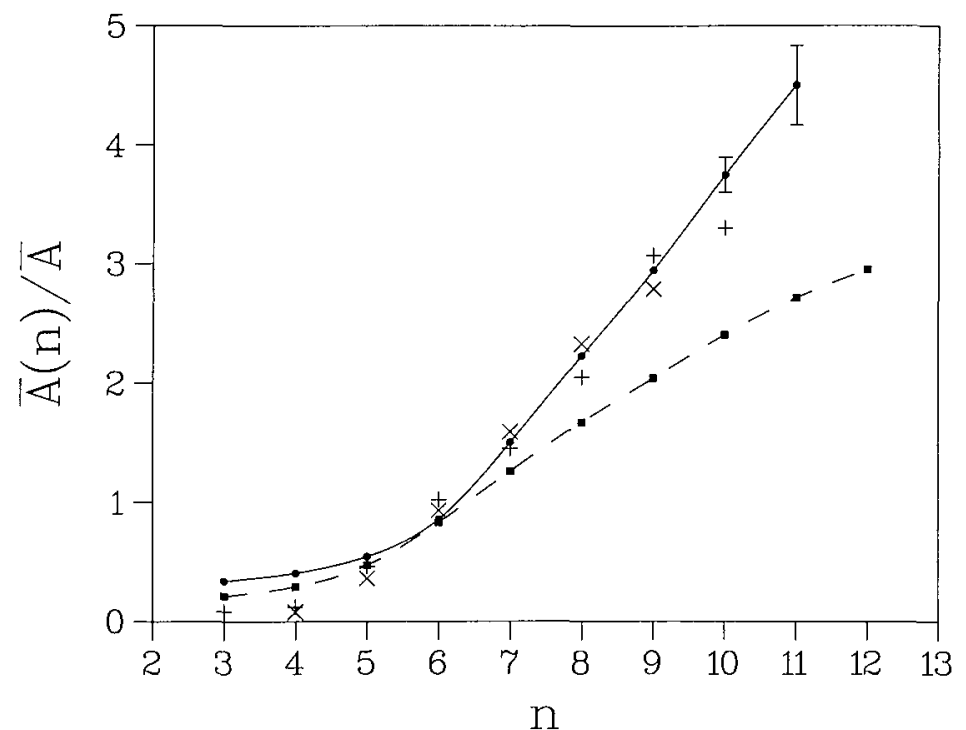

Fig 4 Scaled average area of $n$-sided cells, compating two theories and one expeiıment Solid curve is drawn through points obtained by numerical simulation of the model of section 2 (EIror bars have a length of one standard deviation from the average over 101 uns, for $n \leqslant 9$ this statistical crror is insignificantly small) Dashed curve is Marder's ${ }^{12}$ ) mean-ficld theory Plusses and crosses are measurements by Glazier et $\mathrm{al}^{3}$ ) on a quasi-2D sodp froth, after coarsenıng during 12 hours and 64 hours, respectively

*The imitial state is obtaned by peiforming neighbour-switching T1-piocesses at 1andom on grains in a hexagonal network with periodic boundary conditions - until $40 \%$ hexagonal cells have remained, initially the areas are uncorrelated with the number of sides, and are distributed with a standard deviation of $60 \%$ of the average These initicl conditions correspond to case D in ref 8

${ }^{* *} \mathrm{Eq}(310)$ predicts an asymptotic slope equal to the rate coefficient $\gamma$, defined in eq (3 3b) We find $\gamma \approx 17$ in the simulation, while the slope in fig 4 is only about 07 
The large- $n$ behavior found here agrees with that observed experimentally by Glazier et al. ${ }^{3}$ ) in a quasi-2D soap froth. In fig. 4 measurements at two different times in the experiment are shown. These two sets of data are roughly equivalent, consistent with the existence of a scaling regime. Although for $n>6$ our simulation agrees quite well with this experiment, the calculated average area of 3- and 4-sided cells is considerably larger than observed. A possible cause of this discrepancy is our neglect of the neighbour-switching T1-process (see section 2), which would provide an additional mechanism by which small cells can lose sides before disappearing. Finally, we present a comparison with results of Sahni et al. ${ }^{4}$ ) from a simulation of the Potts lattice model for grain growth in a $2 \mathrm{D}$ polycrystalline network* ${ }^{*}$. Since these authors average the grain "radius" $R \equiv \sqrt{A / \pi}$, rather than its area, we have plotted their data in a separate figure (fig. 5), together with our results for $\bar{R}(n) / \bar{R}$. The differences are comparable to those with the soap froth data. Note also that, of the two empirical rules, the perimeter law $\bar{R}(n)=c_{1}+c_{2} n$ describes the shape-size correlation somewhat better than Lewis' law $\bar{A}(n)=c_{1}+c_{2} n$.

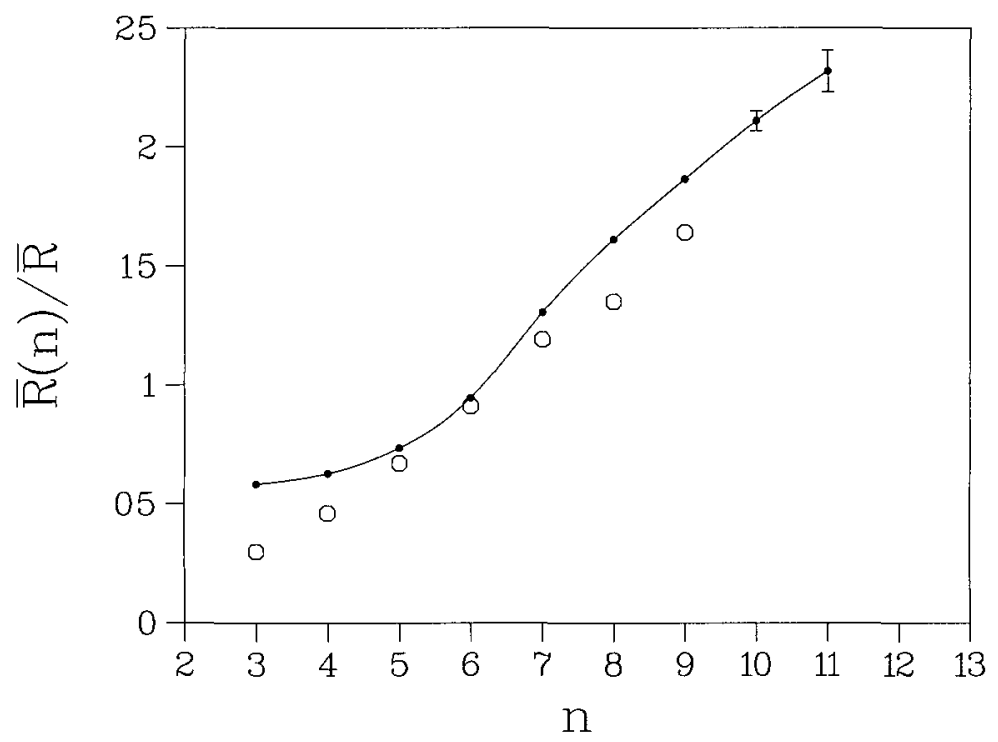

Fig 5 Scaled average radius of $n$-sided cells, comparison of data from the $2 \mathrm{D}$ grain growth simulation by Sahn et $\mathrm{al}^{4}$ ) (circles) with our results (solid curve, error bars as in fig 4)

*An application of this lattice model to $2 \mathrm{D}$ soap froths, and also a contınuum version, have becn studied by Weane and coworkers ${ }^{15}$ ) Their results for the shape-size correlation are similar to those of Sahnı et dl 


\section{Concluding remarks}

An analytical and a numerical study of the shape-size correlation in 2D soap froths (S) and polycrystalline networks (P) have been presented. The results are in general agreement with experimental $\mathrm{data}^{3}$ ) for case $\mathrm{S}$ and with a lattice model simulation ${ }^{4}$ ) for case $P$-although a discrepancy for few-sided cells remains. We conclude with the following remarks.

i) The similarity for the data in cases $\mathrm{S}$ and $\mathrm{P}$ strongly suggests that the shape-size correlation in these two systems has the same origin. Our analysis identifies this common origin as von Neumann's theorem (eq. 2.3), which correlates the growth rate of a cell's area with its number of sides, and holds for both cases $S$ and $P$. An analytic calculation (section 3) shows explicitly that a dynamics based on von Neumann's theorem (plus a diffusion equation for changes in the number of sides) leads to correlations of the type observed.

ii) The shape-size correlation is not fully described by either Lewis' law or the perimeter law. In fact, even the highly simplified treatment of section 3 predicts a rather complicated dependence of $\bar{A}(n)$ on $n$ (eq. (3.10)), starting off quadratically for small $n$ and then reaching a linear dependence for large $n$. In contrast, Rivier's ${ }^{5}$ ) maximum entropy argument for the grain growth problem leads to the perimeter law for all $n$. As an empirical rule, the perimeter law is quite good (cf. fig. 5). However, the fundamental significance attached to this rule by Rivier is not supported by our analysis of the equations of motion.

iii) In the long-time limit, the scaled average $\bar{A}(n) / \bar{A}$ is found to be time independent. This is consistent with a consequence of the scaling hypothesis that the (normalized) distribution of areas and number of sides is time invariant when expressed as a function of $A / \bar{A}(t)$ (eq. (3.3a)). In the literature on the soap froth problem this has been a controversial issue: Early experiments showed no sign of a scaling regime ${ }^{9,10}$ ). In ref. 11 we have proposed a theory for this anomalous non-scaling behavior, in which the many-sided shape of large soap cells is attributed to the relatively low surface energy of such nearly circular cells. The new experiments by Glazier et $a .^{3}$ ), however, disagree with ref. 11 - and we now believe that the surface energy mechanism plays only a minor role in the development of the shape-size correlation. The point is, as argued by Marder $^{12}$ ), that the soap-film network is highly constrained in its movements and cannot easily reach an energetically more favourable structure. Marder's calculations show convincingly that these new experiments can be explained without considering the surface energy mechanism (and also suggest that the anomalous broadening of the scaled distribution function seen in the previous experiments was a transient effect). In this connection we note that the validity of a second consequence of the scaling hypothesis, viz. the linear growth law (3.3b), remains to be established 
experimentally, although theoretically there is considerable evidence in favour, see refs. 8 and 12 for further discussion.

iv) Our analysis is based on von Neumann's theorem (eq. (2.3)), and thus limited to two dimensions. Measurements on 2D sections of 3D polycrystalline materials show a shape-size correlation which is remarkably sumilar to that found in the simulation of $2 \mathrm{D}$ grain growth in ref. 4. This remains to be explained. The mechanism of the correlation will be the same in $3 \mathrm{D}$ as in $2 \mathrm{D}$ - provided that grains with many faces have a concave surface, while those with few faces are convex. (This seems indeed to be the case ${ }^{16}$ ).) Many-faced grains, then, would grow at the expcnse of few-faced ones, leading to a dynamical shape-size correlation in three just as in two dimensions.

\section{Acknowledgement}

It is an honour to dedicate this paper to my teacher, Professor P. Mazur.

\section{References}

1) Sec the icview atticle by D Wearie and N Rivici, Contemp Phys 25 (1984) 59

2) F T Lewis, Andtomical Record 38 (1928) 341

3) J A Glazier, S P Gross, and J Stavans, Phys Rev A 36 (1987) 306

4) PS Sahn1, G S Gtest, M P Anderson and D J Siolovitz, Phys Rev Lett 50 (1983) 263, Acta Mctall 32 (1984) 783. 793

PS Sahnı, D J Srolovitz, G S Gicst, M P Anderson and S A Satran, Phys Rev B 28 (1983) 2705

5) N Rivie1, Philos Mag B 52 (1985) 795

6) J von Neumann, in Metal Interfaces, C Herring, ed (Amenican Society for Metals, Cleveland, 1952), p 108

7) WW Mullins, J Appl Phys 27 (1956) 900

8) CW J Beenakkeı, to be published

9) D A Abodv, Mctallography 13 (1980) 43

10) D Wearie and I P Kermode, Philos Mag B 47 (1983) L29

11) CW J Becnakker, Phys Rev Lett 57 (1986) 2454

12) M Marder, Phys Rev A 36 (1987) 438

13) S M Allen and J W Cahn, Acta Mctall 27 (1979) 1085

14) PM Morsc and H Feshbach, Methods of Theotetical Physics (McGidw-Hill, New York, 1953), vol 1, p 530

15) D Wealle and J P Kcimode, Phulos Mag B 48 (1983) 245, 50 (1984) 379

J We cheit, D Weare and J P Kermode, Philos Mag B 53 (1986) 15

16) F N Rhunes and K R Claig, Metall Tians 5 (1974) 413 